\title{
REDSHIFTS OF FAINT BLUE GALAXIES FROM GRAVITATIONAL LENSING
}

\author{
MATTHIAS BARTELMANN ${ }^{1,2}$ AND RAMESH NARAYAN ${ }^{1}$ \\ ${ }^{1}$ Harvard-Smithsonian Center for Astrophysics \\ 60 Garden Street, Cambridge, MA 02138, USA, and \\ ${ }^{2}$ Max-Planck-Institut für Astrophysik \\ Postfach 1523, 85740 Garching, Germany
}

\section{Introduction}

The possibility to reconstruct cluster mass distributions from their gravitational lensing effects on background sources is now firmly established and is discussed in a number of other contributions to these proceedings. Here, we want to suggest a new method, the lens parallax method (Bartelmann \& Narayan 1995), to infer the redshifts of the background sources, which are in general too faint to allow spectroscopy. The method is based on gravitational lensing, and apart from arclet redshifts it yields calibrated, accurate cluster reconstructions. The key idea is simple and rests on three facts: (1) The strength of lensing effects increases with source redshift, if the lens redshift is kept fixed. (2) The surface brightness of the arclet sources steeply decreases with redshift $z$. Neglecting spectral effects, the surface brightness is $\propto(1+z)^{-4}$. (3) The surface brightness of the sources is invariant to lensing. In combination, these facts imply that lensing effects become stronger with decreasing surface brightness, so that relative distances of arclet sources can be inferred from the variation of lensing effects with the surface brightness of lensed sources. If the redshift of the brightest sources can be measured spectroscopically, the relative distances can be calibrated and the average redshift as a function of surface brightness can be found.

Conversely, knowledge of the source redshifts removes an uncertainty from cluster reconstructions, which usually assume the sources to be all at one redshift. All reconstructions based on image distortions alone suffer from the so-called mass-sheet degeneracy (Falco et al. 1985; Gorenstein 
et al. 1988; Kaiser \& Squires 1993; Schneider \& Seitz 1995), which states that the surface-mass density of the lens can only be reconstructed up to a one-parameter family of transformations. Cluster masses can be reliably determined only if this degeneracy is broken. We suggest including the magnification of the arclet sources in the reconstruction algorithm to eliminate the mass-sheet degeneracy and obtain accurate cluster masses (see also Broadhurst et al. 1995).

The results of numerical simulations designed to test the algorithm we suggest are promising. Using a sample of 12 numerically simulated clusters and a realistic model for the intrinsic source properties, we show that source redshifts can be inferred with an accuracy of $\Delta z \sim 0.1$, and that the masses of the lensing clusters can be reconstructed to within $\pm 5 \%$.

\section{Outline of the Method}

We require the following observational data, which have to be obtained in a sample of cluster fields and a number of empty control fields: The ellipticities $\varepsilon$ of the sources, their surface brightnesses $S$, and their sizes $R$. In the following, primed symbols refer to quantities obtained in control fields. The surface brightnesses are sorted into bins $S_{i}$. The data in the cluster fields depend on position and thus have to be sorted into spatial bins $\vec{x}_{j}$, while the control data should be spatially homogeneous. We thus start from the data sets

- $\varepsilon^{\prime}\left(S_{i}\right)$ and $R^{\prime}\left(S_{i}\right)$, the ellipticity and the size of the sources as a function of their surface brightness in the control fields; and

$-\varepsilon\left(\vec{x}_{j}, S_{i}\right)$ and $R\left(\vec{x}_{j}, S_{i}\right)$, the same properties of the arclets in the cluster fields.

The $\varepsilon^{\prime}\left(S_{i}\right)$ are used to find the intrinsic spread of ellipticities to calibrate a major source of noise in the cluster reconstructions. The sizes are combined to form the quantity

$$
r_{i j} \equiv r\left(\vec{x}_{j}, S_{i}\right) \equiv\left(\frac{R^{\prime}\left(S_{i}\right)}{R\left(\vec{x}_{j}, S_{i}\right)}\right)^{2},
$$

which estimates the inverse magnification at position $\vec{x}_{j}$ for sources with surface brightness $S_{i}$.

For linear lenses which satisfy $\kappa \ll 1$ and $|\gamma| \ll 1$, the ellipticities estimate the shear and the sizes estimate the magnification,

$$
\begin{aligned}
\varepsilon_{i j} & \equiv \varepsilon\left(\vec{x}_{j}, S_{i}\right) \approx \gamma\left(\vec{x}_{j}, S_{i}\right) \equiv \gamma_{i j} \\
r_{i j} & \approx 1-2 \kappa\left(\vec{x}_{j}, S_{i}\right) \equiv 1-2 \kappa_{i j} .
\end{aligned}
$$


Their redshift dependence is expressed by their dependence on surface brightness. We describe it by distance factors $d_{i}$ to relate the data in the surface-brightness bins $S_{i}, i \geq 2$, to the (brightest) bin $S_{1}$,

$$
(\kappa, \gamma)_{i j}=(\kappa, \gamma)_{1 j} \cdot d_{i}
$$

The $d_{i}$ constitute a "distance ladder" for the sources which depends on the adopted cosmological model. Specifying $\Omega$ and $\Lambda$, the $d_{i}$ can be converted to redshifts once the redshift corresponding to $S_{1}$ is measured. From each spatial bin, we obtain two independent estimates for the $d_{i}$,

$$
d_{i} \approx \frac{\varepsilon_{i j}}{\varepsilon_{1 j}} \approx \frac{1-r_{i j}}{1-r_{1 j}}
$$

which can be weighted by their respective estimated accuracies and combined. Conversely, the ellipticities and sizes in each surface-brightness bin provide estimates for convergence and shear according to (2),

$$
\gamma_{1 j} \approx \frac{\varepsilon_{i j}}{d_{i}} \quad, \quad \kappa_{1 j} \approx \frac{1-r_{i j}}{2 d_{i}}
$$

which can equally well be combined.

If the cluster lens is nonlinear, the algorithm becomes somewhat more complicated. Then, ellipticities and sizes measure combinations of convergence and shear rather than each quantity separately,

$$
\varepsilon_{i j}=\frac{\gamma_{i j}}{1-\kappa_{i j}} \quad, \quad r_{i j}=\left(1-\kappa_{i j}\right)^{2}-\gamma_{i j}^{2}
$$

(cf. Kochanek 1990; Miralda-Escudé 1991; Schneider \& Seitz 1995; Kaiser 1995). Since both $\kappa$ and $\gamma$ are initially unknown, the algorithm has to proceed iteratively, but the principle remains as sketched above. The data, $\varepsilon_{i j}$ and $r_{i j}$, are used to iteratively reconstruct the distance factors $d_{i}$ and to build up estimates for $\gamma$ and $\kappa$ of the clusters involved.

In the linear as in the nonlinear case, the reconstructed $\gamma$ can be transformed into $\kappa$, however not uniquely because of the mass-sheet degeneracy. The direct estimate of $\kappa$ from the image magnifications is fairly inaccurate because of the large variance of intrinsic source sizes with given surface brightness (cf. the lower right frame of figure 1). The convergence reconstructed from $\gamma$, however, can be calibrated by the direct estimate of $\kappa$ obtained from the image sizes. This breaks the mass-sheet degeneracy and allows to unambiguously select that cluster model which fits both ellipticities and sizes best, given the data and their errors. 

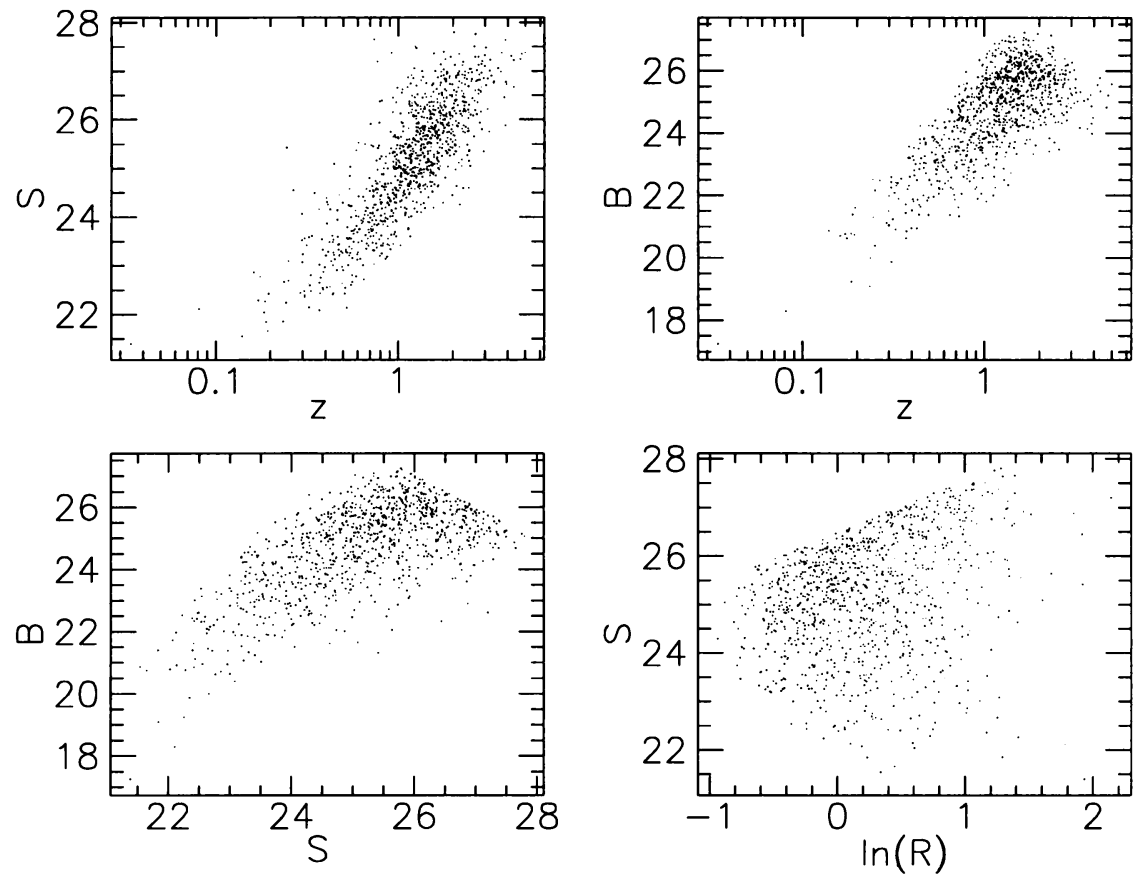

Figure 1. Scatter plots showing the distribution of "observed" properties of our simulated sources. Upper left frame: Surface brightness $S$ in mag $\operatorname{arcsec}^{-2}$ vs. redshift $z ; U p p e r$ right frame: Blue magnitude $B$ vs. $z$; Lower left frame: $B$ vs. $S$; Lower right frame: $S$ vs. size $R$. Sharp boundaries in the plots reflect the detection criterion.

\section{Numerical Model}

To test the feasibility of the proposed algorithm, we use a sample of 12 cluster models simulated in the CDM cosmogony, with $\Omega_{0}=1$ and $\Lambda=0$; see Bartelmann et al. (1995) for a detailed description. The clusters are all at about the same redshift, $z_{\mathrm{d}} \sim 0.35$, and they span a broad mass range. Their velocity dispersions $\sigma_{v}$ fall within $[950,1550] \mathrm{km} \mathrm{s}^{-1}$.

Our model for the sources is guided by the observation that there seems to be little galaxy evolution in red light, although the galaxy number counts evolve significantly in blue light (e.g. Broadhurst et al. 1988; Colless et al. 1990; Colless et al. 1993; Lilly et al. 1991; Lilly 1993; Crampton et al. 1994; Lilly et al. 1995; Koo \& Kron 1992) We adopt the view that the blue light comes from star-forming regions in otherwise normal galaxies. Consequently, we assume a constant comoving number density and a Schechter luminosity function with constant parameters. We further choose their surface-brightnesses, their colors, and their ellipticities from distribution functions which well resemble observational data. The sources are subject to a detection criterion which depends on both surface brightness 

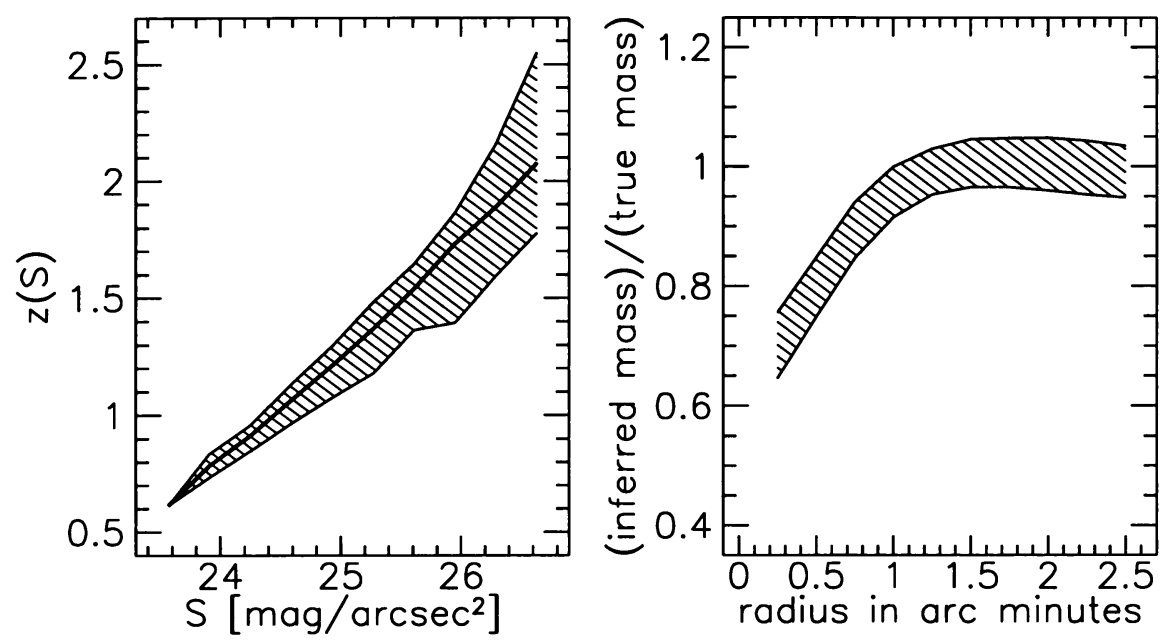

Figure 2. Left frame: Recovered source redshift as a function of surface brightness. The hatched region is centered on the average redshift, and its width corresponds to the 1- $\sigma$ error obtained from 12 cluster and control fields. The heavy line shows the intrinsic source redshift. Right frame: Cumulative reconstructed cluster masses relative to the true masses. While smoothing of the data broadens cluster peaks close to the center and causes the mass underestimate, the reconstructed mass agrees with the true mass to within $\pm 5 \%$ at the field boundary.

and magnitude. The "observed" properties of the simulated sources closely match the observations (cf. figure 1).

\section{Results}

The simulated sources are "observed" in the 12 cluster fields, where they are lensed, and in an equal number of control fields. The simulated data are processed with the iterative algorithm described before. We show the results for the recovered source redshifts and for the reconstructed cluster masses in figure 2.

The left frame shows the source redshifts as a function of surface brightness. The hatched region is centered on the average redshift recovered and its width is the $1-\sigma$ error from the 12 cluster fields. The heavy line represents the intrinsic redshift distribution. The plot shows that the average source redshift is well recovered with an error of $\Delta z \sim 0.1$ at intermediate redshifts. The right frame of the figure displays the cumulative reconstructed mass fraction of the cluster lenses, i.e. the ratio between the reconstructed and the true cluster mass within a given radius. Smoothing of the simulated data broadens the central cluster peaks and causes the mass underestimate within $\sim 1^{\prime}$, but towards the boundary of the field the reconstructed mass equals the true mass to within $\pm 5 \%$ accuracy. 
Summarizing, we find that our simulations indicate that the proposed iterative algorithm succeeds in reconstructing arclet source redshifts and cluster mass distributions to very satisfactory accuracy. The inclusion of the source magnifications breaks the mass-sheet degeneracy inherent in all cluster reconstructions based on image distortions alone and allows for accurate, unambiguous cluster mass determinations.

The lens parallax method can be extended in several ways. First, additional information about the sources can incorporated. The most obvious choice are their colors which are also invariant to lensing. Second, the conversion of distance factors to redshifts depends on the cosmological parameters chosen. Possible inconsistencies between the source redshifts derived from cluster samples with different lens redshifts can be used to constrain especially the cosmological constant $\Lambda$ to which lensing is particularly sensitive (Turner 1990; Kochanek 1993). Third, it can be tested whether a single lens is responsible for the image distortions. If several lenses at different redshifts contribute, the principal axis of the shear in a given spatial bin should change its direction with redshift and thus with the surface brightness of the sources.

This work was supported in part by NSF grant AST 9423209 and by the Sonderforschungsbereich SFB 375-95 of the Deutsche Forschungsgemeinschaft.

\section{References}

Bartelmann, M., \& Narayan, R., 1995, ApJ, in press (Sep. 20 issue)

Koo, D. C., \& Kron, R. G. 1992, ARA\&A, 30, 613

Bartelmann, M., Steinmetz, M., \& Weiss, A., 1995, A\&A, 297, 1

Broadhurst, T. J., Ellis, R. S., \& Shanks, T., 1988, MNRAS, 235, 827

Broadhurst, T. J., Taylor, A. N., \& Peacock, J. A., 1995, ApJ, 438, 49

Colless, M. M., Ellis, R. S., Taylor, K., \& Hook, R. N., 1990, MNRAS, 244, 408

Colless, M. M., Ellis, R. S., Broadhurst, T. J., Taylor, K., \& Peterson, B. A., 1993, MNRAS, 261, 19

Crampton, D., Morbey, C. L., Le Fevre, O., Hammer, F., Tresse, L., Lilly, S. J., \& Schade, D. J., 1994, SISSA preprint astro-ph/9410041

Falco, E. E., Gorenstein, M. V., \& Shapiro, I. I., 1985, ApJ, 289, L1

Gorenstein, M. V., Falco, E. E., \& Shapiro, I. I., 1988, ApJ, 327, 693

Kaiser, N., 1995, ApJ, 493, L1

Kaiser, N., \& Squires, G., 1993, ApJ, 404, 441

Kochanek, C. S., 1990, MNRAS, 247, 135

Kochanek, C. S., 1993, ApJ, 419, 12

Koo, D. C., \& Kron, R. G. 1992, ARA\&A, 30, 613

Lilly, S. J., 1993, ApJ, 411, 501

Lilly, S. J., Cowie, L. L., \& Gardner, J. P., 1991, ApJ, 369, 79

Lilly, S. J., Tresse, L., Hammer, F., Crampton, D., \& Le Fevre, O., 1995, ApJ, submitted Miralda-Escudé, J., 1991, ApJ, 370, 1

Schneider, P., \& Seitz, C., 1995, A\&A, 294, 411

Turner, E. L., 1990, ApJ, 365, 43 